\title{
Translation
}

\section{Genauigkeit und Geschwindigkeit der Proteinsynthese}

WOLFGANG WINTERMEYER, MARINA V. RODNINA

MAX-PLANCK-INSTITUT FÜR BIOPHYSIKALISCHE CHEMIE, GÖTTINGEN

Genauigkeit und Geschwindigkeit der Proteinsynthese sind fundamentale Parameter für die Biologie von Zellen. Zur Optimierung benutzen Ribosomen komplexe Mechanismen der Substratselektion, vor allem induced fit und kinetische Partitionierung.

Accuracy and speed of protein synthesis are fundamental parameters for the biology of cells. For optimization, ribosomes use complex mechanisms of substrate selection, in particular induced fit and kinetic partitioning.

\section{Mechanismus der Proteinsynthese}

- Die schnelle und genaue Auswahl des korrekten Substrats ist für Enzyme mit einem einzigen Substrat in der Regel kein Problem, da hinreichend viele spezifische Wechselwirkungen zwischen Enzym und Substrat ausgebildet werden können. Dagegen müssen Polymerasen in jeder Runde der Elongation aus verschiedenen, chemisch und strukturell ähnlichen Substraten das jeweils richtige auswählen. Obwohl dabei eine einzige Wasserstoffbrücke mehr oder weniger über korrekt und nicht-korrekt entscheiden kann, müssen Genauigkeiten von $10^{4}$ bis $10^{8}$ oder

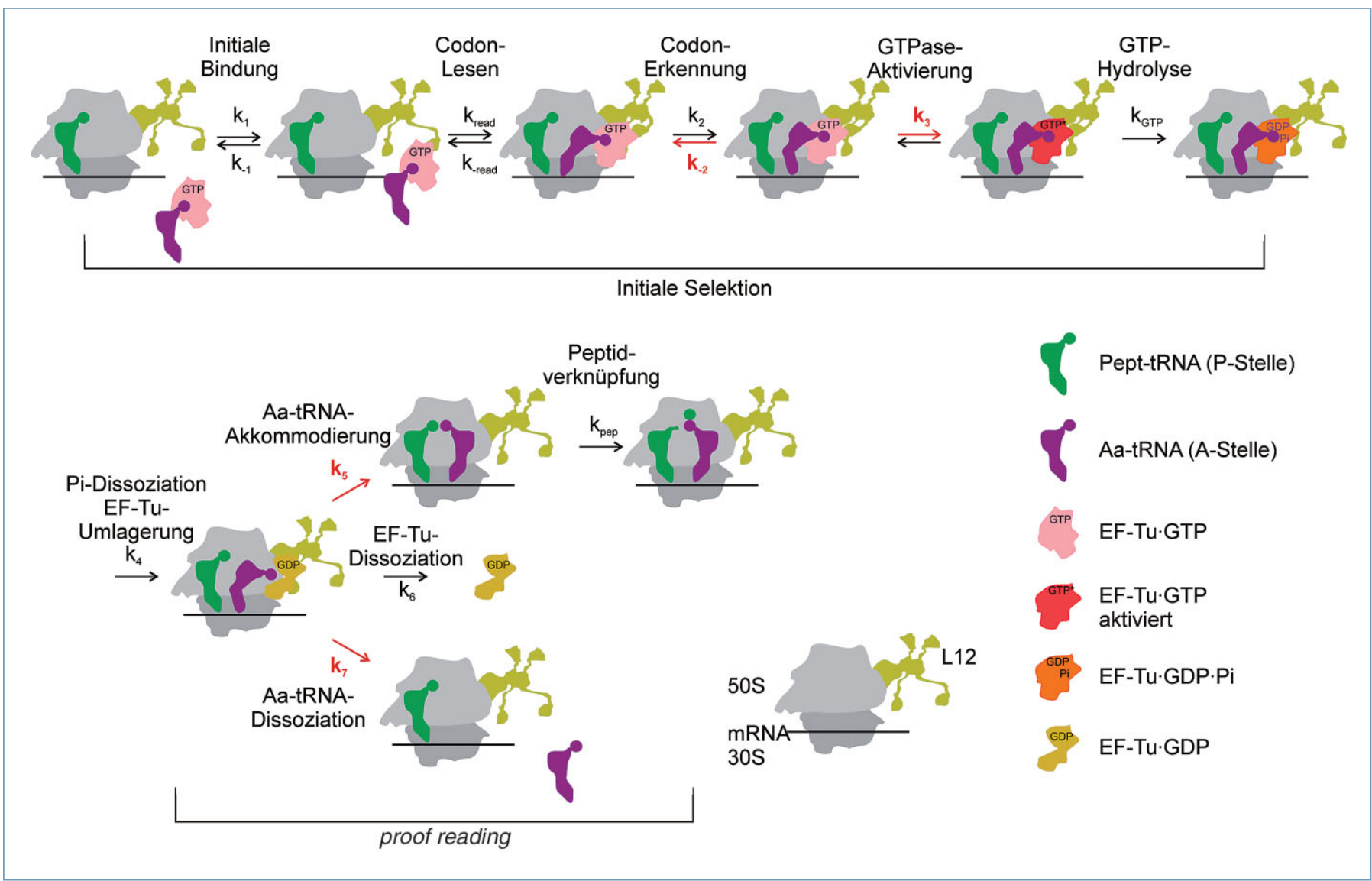

$\Delta$ Abb. 1: Kinetischer Mechanismus der Bindung von Aa-tRNA und Peptidverknüpfung. Die Reaktionsgeschwindigkeitskonstanten $k_{1}$ bis $k_{7}$ und $k_{-1}$ bis $\mathrm{k}_{-7}$ wurden durch schnelle kinetische Messungen (stopped-flow bzw. quench-flow) unter pseudo-first-order-Bedingungen bestimmt. Die chemischen Schritte (GTP-Hydrolyse, Peptidverknüpfung) sind intrinsisch schnell, jedoch kinetisch von der jeweils vorhergehenden Umlagerung (GTPase-Aktivierung, $\mathrm{k}_{3}$, bzw. Akkommodierung, $\mathrm{k}_{5}$ ) bestimmt. Die für die Substratselektion in der initialen Selektion $\left(\mathrm{k}_{-2}, \mathrm{k}_{3}\right)$ und im proofreading $\left(\mathrm{k}_{5}, \mathrm{k}_{7}\right)$ wichtigen Schritte sind rot markiert. Die 30S- und 50S-Untereinheiten des Ribosoms sind dunkel- bzw. hellgrau dargestellt. 

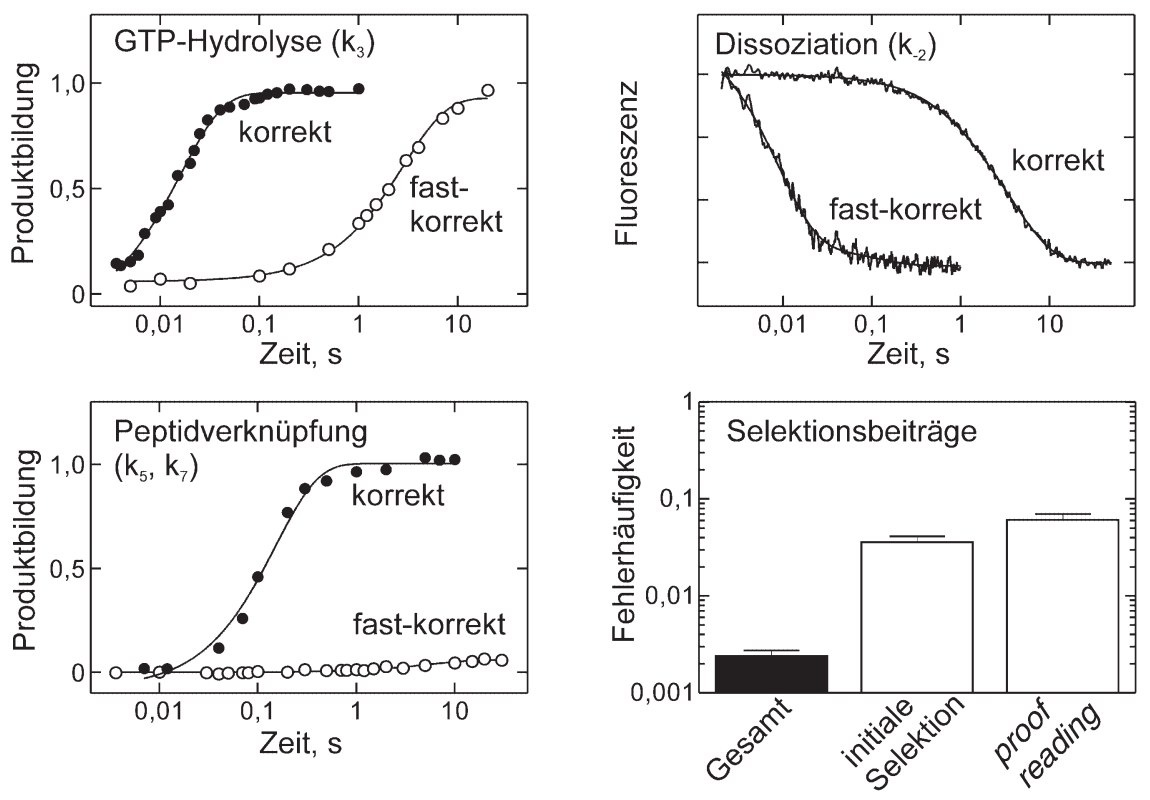

$\Delta$ Abb. 2: Beiträge zur Substratselektion. Kinetische Messungen mit EF-Tu.GTP.Phe-tRNA und Ribosomen mit den Codons UUU (korrekt) oder CUC (fast-korrekt) in der A-Stelle (HiFi-Puffer bei $20^{\circ} \mathrm{C}$ ) [4]. GTP-Hydrolyse und Peptidbildung wurden mit radioaktiv markierten Substraten gemessen, die Dissoziation mit fluoreszenzmarkierter tRNA. Der Gesamtfehler ist das Produkt der Fehlerhäufigkeiten aus initialer Selektion und proofreading. Die aus den kinetischen Konstanten berechneten Fehlerhäufigkeiten [4] stimmen mit den direkt gemessenen gut überein.

höher erreicht werden. Die Lösung ist ein komplexer Bindungsmechanismus mit induced fit-Komponenten und - etwa bei DNAPolymerasen - die Einführung von Korrekturschritten. Das gilt auch für Ribosomen, die Protein-Polymerasen der Zelle.

Ribosomen wählen ihre Substrate, die in Form von aktivierten Aminosäuren als Aminoacyl-tRNAs (Aa-tRNAs) vorliegen, entsprechend der Komplementarität der Anticodon-Sequenz zum Codon-Triplett auf der mRNA aus. Aa-tRNAs binden an das Ribosom in Form sehr stabiler Komplexe mit einem Elongationsfaktor (EF-Tu in Bakterien) und GTP (Ternärkomplex). In Abhängigkeit von der Passung des Anticodons der tRNA im Ternärkomplex an das Codon, das gerade übersetzt werden muss, erfolgen die weiteren Schritte. Bei Erkennung eines vollständig komplementären Codons, also der Ausbildung von drei Basenpaaren zwischen Anticodon und Codon, wird die Hydrolyse von GTP durch EF-Tu aktiviert. Dies führt dazu, dass die Aa-tRNA aus dem Komplex mit EF-Tu freigesetzt wird und in das aktive Zentrum des Ribosoms binden kann, wo es zur Peptidverknüpfung kommt (Abb. 1). Dieser - auf den ersten Blick recht aufwendig wirkende - Mechanismus der Substratbindung ermöglicht dem Ribosom die hinreichend genaue Unterscheidung zwischen korrekten (cognate), inkorrekten (non-cognate) und fastkorrekten (near-cognate, zwei statt drei Basenpaare zwischen Anticodon und Codon) Ternärkomplexen bei gleichzeitig hoher Geschwindigkeit. Die beobachteten Fehlerhäufigkeiten des Aminosäureeinbaus in der Proteinsynthese liegen bei $1: 1.000$ bis 1:10.000, je nach Codon und Codon-Kontext, die Geschwindigkeit bei zehn bis 20 pro Sekunde pro Ribosom. Im Folgenden werden die Mechanismen beschrieben, mit denen dies erreicht wird.

\section{Kinetische Selektion: die Rolle der Vorwärtsreaktionen}

Die Genauigkeit der Aa-tRNA-Selektion am Ribosom wird in zwei Phasen kontrolliert (Abb. 1, [1]): während der Bindung bzw. Dissoziation der Ternärkomplexe vor der GTPHydrolyse (initiale Selektion) und bei der Akkommodierung bzw. Dissoziation der Aa-tRNAs nach der GTP-Hydrolyse (proofreading). Eine weitere Korrekturmöglichkeit, die allerdings erst nach einem Fehleinbau greift und zum Verlust des bereits synthetisierten Peptids führt, ist die Hydrolyse inkorrekt gebildeter Peptidyl-tRNAs am Ribosom durch Terminationsfaktoren [2]. Die beobachtete Häufigkeit des Einbaus falscher Aminosäu- ren hängt einmal von der Effizienz dieser Selektions-bzw. Korrekturmechanismen, zum anderen von der Häufigkeit der jeweils korrekten Aa-tRNAs relativ zu den fast-korrekten Konkurrenten.

Die kinetische Analyse hat vier Elementarreaktionen identifiziert, deren Geschwindigkeitskonstanten für korrekte und fast-korrekte Ternärkomplexe bzw. Aa-tRNAs verschieden sind, während die anderen Teilschritte sich kaum unterscheiden (Abb. 2, [3, 4]). Die ersten Schritte, initiale Bindung und Codon-Erkennung, sind unabhängig vom Codon, da die Reaktionsgeschwindigkeitskonstanten für korrekte und fast-korrekte Paarung gleich sind $\left(\mathrm{k}_{1}, \mathrm{k}_{-1}, \mathrm{k}_{2}\right)$. Dagegen gibt es große Unterschiede in $\mathrm{k}_{-2}$, das heißt der fastkorrekte Ternärkomplex dissoziiert einige 100-mal schneller als der korrekte. Die folgenden Schritte der GTPase-Aktivierung $\left(\mathrm{k}_{3}\right)$ und GTP-Hydrolyse sind im Komplex mit korrekter Anticodon-Codon-Paarung mehr als 500-mal schneller als mit fast-korrekter, und die Akkommodierung $\left(\mathrm{k}_{5}\right)$ der korrekten Aa-tRNA ist deutlich schneller als die der fastkorrekten, die dafür wesentlich schneller dissoziiert $\left(\mathrm{k}_{7}\right)$. Andererseits ist die GTP-Hydrolyse vergleichbar schnell für verschiedene korrekte Anticodon-Codon-Paarungen [5]; Ähnliches gilt für die Peptidverknüpfung [6]. Diese gleichförmige Reaktivität wird erreicht durch den Ausgleich der unterschiedlich starken Einflüsse des jeweiligen Aa-Rests durch evolutive Anpassung der tRNAs in Sequenz und Struktur [5]. Dies scheint auch für die (viel langsamere) GTP-Hydrolyse bzw. Dissoziation bei fast-korrekter Paarung zu gelten [4].

Der Hauptdiskriminierungsfaktor ist demnach die kinetische Partitionierung zwischen GTPase-Aktivierung $\left(\mathrm{k}_{3}\right)$ und Dissoziation des Ternärkomplexes $\left(\mathrm{k}_{-2}\right)$ bzw. zwischen Akkommodierung $\left(\mathrm{k}_{5}\right)$ und Dissoziation $\left(\mathrm{k}_{7}\right)$ der Aa-tRNA. Der etwa 1.000-fache Unterschied der Werte für $\mathrm{k}_{-2}$ zeigt, dass der Unterschied der thermodynamischen Stabilitäten der korrekten und fast-korrekten Codon-Erkennungskomplexe, $\Delta \Delta \mathrm{G}^{\circ}$, prinzipiell in etwa ausreichend wäre, die beobachteten Genauigkeiten zu erklären. Es ist jedoch wichtig, sich klarzumachen, dass dieses $\Delta \Delta \mathrm{G}^{\circ}$ nicht unmittelbar für die Selektion wirksam wird, weil das System das Gleichgewicht nicht erreicht und auch nicht erreichen soll, da das viel zu langsam wäre. Der Grund ist, dass im Fall des korrekten Codon-Erkennungskomplexes die Folgereaktionen GTPase-Aktivierung und GTP-Hydrolyse so schnell erfolgen, 
dass der Bindungsschritt nicht equilibrieren kann $\left(\mathrm{k}_{3}>\mathrm{k}_{-2}\right)$. Die kinetische Partitionierung führt demnach dazu, dass der korrekte Komplex mit sehr hoher Präferenz (1.000-fach) in die GTP-Hydrolyse geht, während der fast-korrekte Komplex $\left(\mathrm{k}_{3} \ll \mathrm{k}_{-2}\right)$ vorzugsweise (200-fach) dissoziiert.

Als Konsequenz aus der raschen GTPHydrolyse und der Nicht-Erreichung des Gleichgewichts ist die Michaelis-Konstante $\left(\mathrm{K}_{\mathrm{M}}\right)$ für die Bildung des korrekten CodonErkennungskomplexes viel größer (GröBenordnung $\mu \mathrm{M}$ ) als die im Gleichgewicht gemessene Bindungskonstante (Größenordnung 0,01 $\mu \mathrm{M})$. Die - auf den ersten Blick vielleicht kontra-intuitive - Folge ist, dass die $\mathrm{K}_{\mathrm{M}}$-Werte für korrekte und fastkorrekte Codon-Erkennungskomplexe kaum verschieden sind, obwohl die Affinitäten sich um einen Faktor 400 unterscheiden, sodass die Selektion zwischen korrektem und fast-korrektem Substrat nicht, wie sonst bei den meisten Enzymen, auf den Unterschieden in den Werten für $\mathrm{k}_{\mathrm{cat}} / \mathrm{K}_{\mathrm{M}}$ beruht, sondern dass praktisch nur die Unterschiede in den $\mathrm{k}_{\mathrm{cat}}$-Werten zum Tragen kommen.

\section{Strukturelle Basis der Selektion}

Die initiale Bindung des Ternärkomplexes an das Ribosom ist unabhängig vom Codon und vor allem vermittelt durch Kontakte zwischen EF-Tu und dem ribosomalen Protein L7/12, von dem das Escherichia coliRibosom vier Kopien aufweist. Das anschlieBende Codon-Lesen wird ermöglicht durch strukturelle Fluktuationen der tRNA, insbesondere eine Verbiegung des AnticodonStamms, wodurch das Anticodon das Codon im Decodierzentrum der 30S-Untereinheit erreichen kann, während der Rest des tRNA-Moleküls an EF-Tu auf der 50S-Untereinheit gebunden bleibt (Abb. 3, [7]). Die Ausbildung eines korrekten, vollständig gepaarten Anticodon-Codon-Komplexes führt zu einer Umlagerung im Decodierzentrum, bei der A-1492, A-1493 und G-530 der 16S-rRNA ihre Position verändern und Wechselwirkungen mit dem AnticodonCodon-Duplex ausbilden [8]. In der ersten und zweiten Codon-Position erkennt das Ribosom korrekte Basenpaare aufgrund ihrer Watson-Crick-Geometrie, unabhängig von der Sequenz, während in der dritten Position auch wobble-Basenpaare „erlaubt“ sind. Die Umlagerung im Decodierzentrum wird begleitet von einer globalen Umlage- rung der Kopfdomäne der 30S-Untereinheit und weiteren Umlagerungen des Ribosoms, die letzendlich zur Stimulierung der GTPase-Aktivität von EF-Tu und zur GTP-Hydrolyse führen. Das Ribosom wirkt demnach wie ein Verstärker, der ein relativ schwaches Eingangssignal - die freie Energie der Anticodon-Paarung - in ein wesentlich größeres Signal (GTP-Hydrolyse) umwandelt. Dieses wirkt auf die Konformation von EFTu, die den Zugang des Substrats zum aktiven Zentrum kontrolliert.

Das Decodierzentrum auf der kleinen Untereinheit des Ribosoms ist fast 80 Angström entfernt von der GTP-Bindungstasche von EF-Tu auf der großen Untereinheit, wo die GTP-Hydrolyse stattfindet. Eine wichtige Frage ist demnach, wie die Erkennung eines korrekten Codons sowohl die GTPaseAktivierung als auch die Akkommodierung beschleunigen kann. GTP wird hydrolysiert durch den Angriff eines Wassermoleküls auf das $\gamma$-Phosphat. Den Übergangszustand am Ribosom stabilisiert His-84, das im ungebundenen EF-Tu weg vom hydrolytischen Wasser orientiert und am Zugang zum aktiven Zentrum gehindert ist. Bei der GTPase-Aktivierung bewegt sich His-84 zum $\gamma$-Phosphat hin. Diese Bewegung muss durch Codon-Erkennung ausgelöst werden. Offenbar spielt dabei die schon erwähnte Verzerrung der Aa-tRNA eine wichtige Rolle, da die GTPase-Aktivierung nicht zustande kommt, wenn das tRNA-Molekül nicht intakt ist, und beeinträchtigt wird durch Mutationen, welche die Struktur der tRNA an der Stelle der Verzerrung beeinflussen [9]. Die Kristallstruktur zeigt, dass die relative Orientierung von Aa-tRNA und EF-Tu durch die Verzerrung der tRNA beeinflusst wird [7]. Dies führt zu einer Stabilisierung der katalytisch wirksamen Orientierung von His-84 durch eine Wechselwirkung mit der Sarcin-Ricin-Schleife der 23S-rRNA, die ursächlich für die GTPase-Aktivierung ist.

Die Uniformität der Erkennung von Fehlpaarungen im Anticodon-Codon-Duplex deutet auf einen globalen Mechanismus hin, bei dem alle Konformationsveränderungen, die durch die Erkennung eines korrekten Codons induziert werden (Umlagerung der 30S-Kopfdomäne, Verzerrung der tRNA und Umlagerungen in EF-Tu), essenziell für die genaue Positionierung des aktiven Zentrums von EF-Tu an der SarcinRicin-Schleife sind. In den Fällen, wo ein fast-korrektes Codon erkannt wird, sind offenbar nicht alle diese Elemente ausge- 


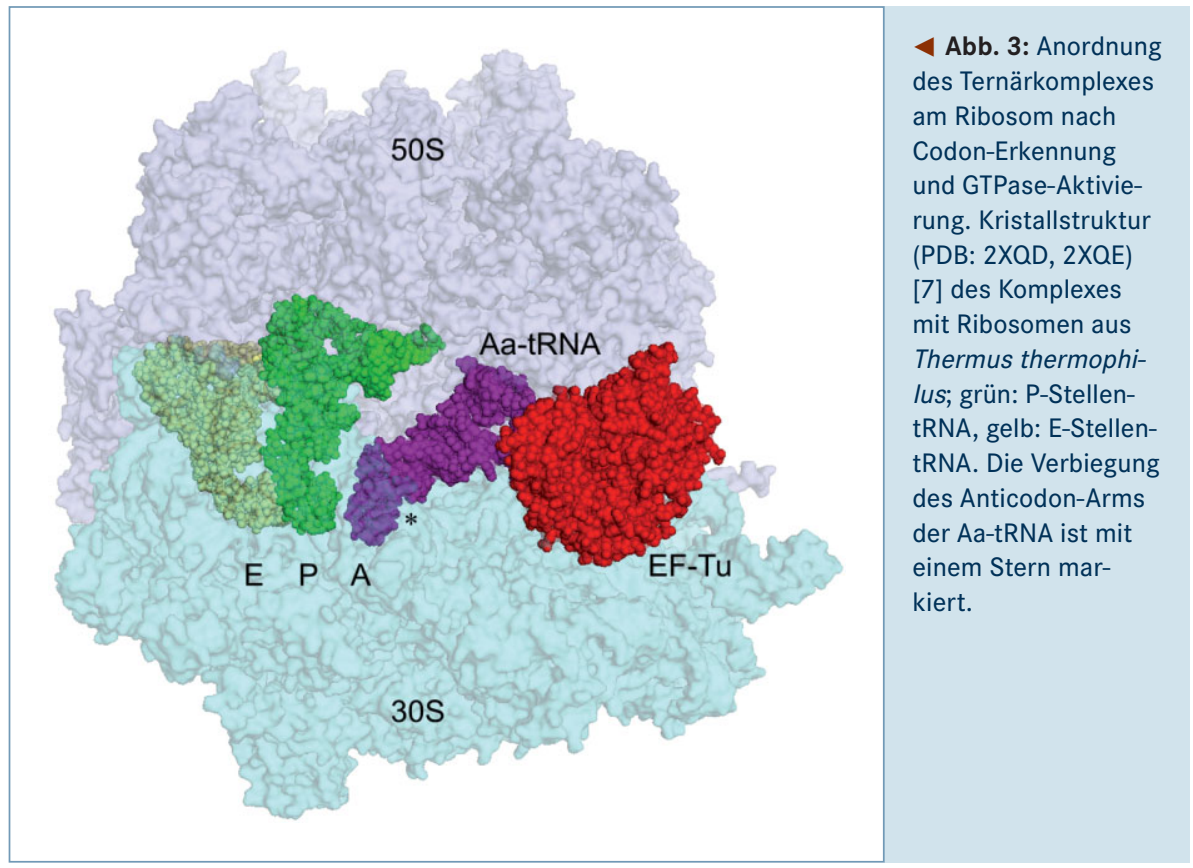

bildet, sodass es trotz des Vorliegens einer verzerrten tRNA-Struktur [10] nicht zur vollen GTPase-Aktivierung kommt.

Im Vergleich zur GTP-Hydrolyse ist die strukturelle Basis der codonspezifischen Beschleunigung der Peptidverknüpfung weniger gut verstanden. Kinetisch wird die Reaktion limitiert durch die Akkommodierung der Aa-tRNA im Peptidyltransferase-Zentrum [6]. Molekulardynamische Berechnungen deuten auf eine schrittweise Akkommodierung hin, bei der sich die tRNA durch einen Korridor konservierter rRNA-Basen bewegt [11]. Die korrekte Ausrichtung des Anticodons im Decodierungszentrum führt $\mathrm{zu}$ schneller Akkommodierung. Offenbar sind die Anticodons inkorrekter tRNAs nicht optimal ausgerichtet, sodass auch die Ausrichtung des Akzeptor-Arms und seine Bewegung durch den Akkommodierungskorridor beeinträchtigt sein könnte. Neben diesen möglichen Beeinträchtigungen an der Eingangspforte könnte eine falsche Ausrichtung des Anticodon-Arms auch die sonstigen Wechselwirkungen zwischen tRNA-Molekül und Ribosom [12] stören und damit die Akkommodierung der fast-korrekten Aa-tRNA behindern und ihre Dissoziation fördern.

\section{Das Ribosom als Ribozym}

Das Ribosom beschleunigt die Peptidverknüpfung um einen Faktor von $10^{7}$ bis $10^{8}$, ist also - wie Ribozyme generell - ein weniger effizienter Katalysator als viele Proteinenzyme, die Beschleunigungen um bis $\mathrm{zu}^{10^{23}}$ fach erreichen. Die intrinsische Geschwindigkeit der Peptidverknüpfung ist kinetisch durch die deutlich langsamere Akkommodierung der Aa-tRNA limitiert. Dies ist vermutlich auch der Grund dafür, dass die RNAbasierte, entropische Katalyse in der Evolution vom präbiotischen Ribozym zum modernen Ribosom beibehalten werden konnte. Dagegen musste die Genauigkeit mit zunehmender Größe und funktioneller Komplexität der Produkte des Translationsapparats erhöht werden. Der wesentliche Schritt war die Kopplung der Substratbindung an die Hydrolyse von GTP durch das Hinzutreten einer GTPase, die Aa-tRNA nur zur Peptidverknüpfung zulässt, wenn die Qualität der Anticodon-Codon-Passung geprüft ist - sogar doppelt, nämlich in der initialen Selektion und im proofreading. Eine wesentliche Rolle spielt dabei das Ribosom selbst, das die korrekte Anticodon-Codon-Paarung erkennt und die dadurch induzierten komplexen Strukturveränderungen zur Aktivierung der

\begin{tabular}{|c|c|c|}
\hline \multicolumn{3}{|l|}{ AUTOREN } \\
\hline & $\begin{array}{l}\text { Marina V. Rodnina } \\
\text { Biologiestudium in Kiew, Ukrai- } \\
\text { ne. } 1989 \text { Promotion. 1990- } \\
1992 \text { Humboldt-Stipendium. } \\
1998 \text { Habilitation; } 1998-2000 \\
\text { Professur. 2000-2008 Lehr- } \\
\text { stuhl Physikalische Biochemie, } \\
\text { Universität Witten/Herdecke. } \\
\text { Seit } 2008 \text { Direktorin am Max- } \\
\text { Planck-Institut für biophysikali- } \\
\text { sche Chemie, Göttingen. }\end{array}$ & $\begin{array}{l}\text { Wolfgang Wintermeyer } \\
\text { Chemiestudium in München. } \\
1972 \text { Promotion. } 1979 \text { Habili- } \\
\text { tation. 1982-1987 Heisen- } \\
\text { berg-Stipendium der DFG. } \\
1987-2009 \text { Lehrstuhl Moleku- } \\
\text { larbiologie, Universität Wit- } \\
\text { ten/Herdecke. Seit } 2009 \text { Max- } \\
\text { Planck-Fellow am Max-Planck- } \\
\text { Institut für biophysikalische } \\
\text { Chemie, Göttingen. }\end{array}$ \\
\hline
\end{tabular}

GTPase von EF-Tu umsetzt - ein Beispiel für ein System, das durch Ko-Evolution optimiert wurde.

\section{Literatur}

[1] Rodnina MV, Wintermeyer W (2001) Fidelity of aminoacyl-tRNA selection on the ribosome: kinetic and structural mechanisms. Annu Rev Biochem 70:415-435

[2] Zaher HS, Green R (2009) Quality control by the ribosome following peptide bond formation. Nature 457:161-166

[3] Pape T, Wintermeyer W, Rodnina M (1999) Induced fit in initial selection and proofreading of aminoacyl-tRNA on the ribosome. EMBO J 18:3800-3807

[4] Gromadski KB, Daviter T, Rodnina MV (2006) A uniform response to mismatches in codon-anticodon complexes ensures ribosomal fidelity. Mol Cell 21:369-377

[5] Ledoux S, Uhlenbeck OC (2008) Different aa-tRNAs are selected uniformly on the ribosome. Mol Cell 31:114-123

[6] Wohlgemuth I, Pohl C, Rodnina MV (2010) Optimization of speed and accuracy of decoding in translation. EMBO J 29:3701-3709

[7] Voorhees RM, Schmeing TM, Kelley AC et al. (2010) The mechanism for activation of GTP hydrolysis on the ribosome. Science 330:835-838

[8] Schmeing TM, Ramakrishnan V (2009) What recent ribosome structures have revealed about the mechanism of translation. Nature 461:1234-1242

[9] Zaher HS, Green R (2009) Fidelity at the molecular level: lessons from protein synthesis. Cell 136:746-762 [10] Mittelstaet J, Konevega AL, Rodnina MV (2011) Distortion of tRNA upon near-cognate codon recognition on the ribosome. J Biol Chem 286:8158-8164

[11] Sanbonmatsu KY, Joseph S, Tung CS (2005) Simulating movement of tRNA into the ribosome during decoding. Proc Natl Acad Sci USA 102:15854-15859

[12] Jenner L, Demeshkina N, Yusupova G et al. (2010) Structural rearrangements of the ribosome at the tRNA proofreading step. Nat Struct Mol Biol 17:1072-1078

Korrespondenzadresse:

Prof. Dr. Marina V. Rodnina

Abteilung für physikalische Biochemie

Max-Planck-Institut für biophysikalische Chemie Am Fassberg 11

D-37077 Göttingen

Tel.: 0551-201-2900

Fax: 0551-201-2905

rodnina@mpibpc.mpg.de 